\title{
Comparison of Spatio-Temporal Parameter and Symmetry during Gait between Healthy EIderly and Young People
}

\author{
Kyue-Nam Park, PT, Ph.D \\ Department of Physical Therapy, College of Medical Science, Jeonju University, Jeonju, South Korea
}

Background Previous studies have examined the gait patterns of healthy elderly people who are able to walk without musculoskeletal pain, but results have been conflicting. Systemic diseases such as diabetes, depression, and hypertension, as well as musculoskeletal system dysfunction, can induce gait deterioration.

Purpose The purpose of the current study was to compare velocity, cadence, step width, stride length, center of pressure (COP) and symmetry between healthy young and elderly people who had not been diagnosed with depression, hypertension, dementia, diabetes, or osteoarthritis.

Study design Cross-sectional study

Methods Twenty-four healthy elderly and twenty healthy young people participated in this study. A force distribution plate was used to obtain data on spatiotemporal parameters, COP, and gait symmetry. The groups were compared using independent $t$-tests.

Results There was no significant difference in walking velocity, step width, stride length, symmetry of step length, foot rotation, length of gait, single support line, or lateral symmetry between the elderly and young groups $(p>0.05)$. However, the elderly group showed higher cadence and larger anterior-posterior sway than the young group (mean difference $=8.98 \mathrm{steps} / \mathrm{min}$, $p<0.01$; and mean difference $=5.88 \mathrm{~mm}, p=0.04$, respectively).

Conclusions The gait patterns of healthy elderly people without depression, hypertension, dementia, diabetes, or musculoskeletal were similar in terms of spatiotemporal parameters and symmetry to those of healthy young people, except for cadence and anterior-posterior sway. Based on these findings, clinicians should inform elderly people about the importance of maintaining fitness to prevent deterioration of gait.

\author{
J Musculoskelet \\ Sci Technol \\ 2021; 5(1): 27-33 \\ Published Online \\ Jun 30, 2021 \\ pISSN 2635-8573 \\ eISSN 2635-8581
}

Article History Received 15 Mar 2021 Revised 13 Apr 2021 (1st) Revised 20 Apr 2021 (2nd) Accepted 20 Apr 2021

\section{CONTACT}

knpark@jj.ac.kr Kyue-Nam Park, Department of Physical Therapy, College of Medical Science, Jeonju University, Jeonju, South Korea

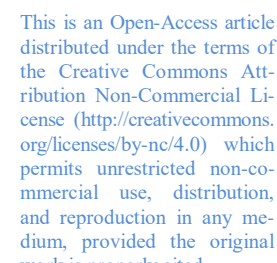
dium, provided the origina work is properly cited.

Key words Aging; Healthy; Spatiotemporal parameter; Symmetry; Walking.

\section{INTRODUCTION}

Elderly people exhibit slower, shorter, and wider steps compared to young people, to increase stability and energy efficiency and avoid falling. ${ }^{1,2}$ Such gait adaptations are related to increased fall risk during daily activities. ${ }^{3}$ Previous studies have suggested that aging is not the main factor causing gait deterioration; rather, a fear of falling is more likely to alter gait patterns.,

Some studies have compared spatiotemporal gait parame- ters between young and elderly populations, but results have been conflicting. A shorter stride length and greater step width have been reported in healthy elderly compared to young populations, during both treadmill and overground walking. ${ }^{6-8}$ However, another study demonstrated similar cadence and step length between a healthy elderly and young group at slow, fast, and preferred walking speeds, although double limb support was increased in the elderly group. ${ }^{9}$ Moreover, the healthy elderly group showed higher cadence than the young group, which was interpreted as a 
compensatory walking strategy, although another study showed no difference in cadence between healthy elderly and young groups at a self-selected walking speed. ${ }^{10,11}$

Gait symmetry has a tendency to decrease with age; this decrease is greater in elderly fallers than in healthy elderly people, and is associated with a low level of independence in daily activities such as feeding, bathing, transfer, and dressing, as well as with continence disorders. ${ }^{12,13}$ Gait symmetry has been investigated to determine the cause of altered gait patterns in elderly populations, but results have been conflicting. ${ }^{2}$ In studies using a trunk accelerometer, elderly people showed less symmetry during gait than younger people, ${ }^{14,15}$ whereas another study showed no significant association between age and gait symmetry within a healthy elderly population. ${ }^{16}$ With regard to the center of mass, or center of pressure (COP), healthy elderly people showed less symmetry of anterior-posterior acceleration at the center of mass compared to young people, but similar symmetry of medio/lateral direction change. ${ }^{17}$

In the previous studies of altered spatiotemporal parameters and gait asymmetry mentioned above, the definition of "healthy" varied, where the criteria included the absence of locomotor system pathologies, no medical history, sufficient mobility and strength of the musculoskeletal system for gait, ability to walk and perform activities of daily living independently, and absence of major cardiovascular, musculoskeletal, and neurological conditions. ${ }^{10,18,19}$ However, diabetes, depression, and dipper hypertension in elderly people can also reduce gait velocity and stride length compared to healthy controls. ${ }^{20-22}$ Thus, in this study, the healthy elderly participants did not have diabetes, depression, hypertension, dementia, or musculoskeletal disorders. The purpose of the study was to compare the walking velocity, cadence, step width, stride length, COP variables, and gait symmetry between pre-screened healthy elderly and young people. We hypothesized that the gait parameters and symmetry of the two groups would be similar.

\section{METHODS}

\section{Participants}

The purpose and procedures of the study were explained to the participants, all of whom signed the approved consent form. This study was approved by the Jeonju University Campus Human Studies Committee. A sample size of at least 16 subjects per group was needed for a power of 0.75 , assuming an effect size of $\mathrm{d}=0.6$ and significance level of $0.05 .^{23}$ We recruited 24 healthy elderly and 20 healthy young people from three local clubs and Jeonju University (Table 1). The inclusion criteria were as follows: aged over 50 years for the elderly and in the 20 s for the young group; able to walk for at least 1 hour, and to step up and down in daily living, without assistance of any kind or musculoskeletal pain in the lower back or lower limbs; no known clinical history of falling or psychiatric, neurological, musculoskeletal, or cardiovascular problems; and not taking any regular medication. All elderly participants were screened by physicians to confirm their self-reported diagnoses and/or prescribed medications; those with depression, hypertension, dementia, diabetes, osteoarthritis, etc. were excluded. All participants preferred to use the right lower limb when initiating gait or kicking a ball; hence, that limb was considered dominant. ${ }^{24,25}$

\section{Instruments}

A Zebris FDM 1.5 force distribution plate (ZEBRIS $\mathrm{GmbH}$, Isny, Germany) was used to measure gait parameters and COP during gait. This measuring plate has 11,264 force sensors and a sampling frequency of $100 \mathrm{~Hz}$. The gait measurement system, comprising a 3-meter-long pressure plate connected to two FDM plates, has high within- and between-day reliability (intraclass correlation coefficients $>$ $0.86) .{ }^{26}$ The system represents the gait pattern as a two- or three-dimensional butterfly graph based on COP trajectories during gait. The gait analysis system takes account of foot

Table 1. Subject's characteristics

\begin{tabular}{cccc}
\hline Characteristics & Elderly $(\mathrm{N}=24)$ & Young $(\mathrm{N}=20)$ & $p$ \\
\hline Gender $(\mathrm{M} / \mathrm{F})$ & $3 / 21$ & $6 / 14$ & - \\
Age (years) & $59.04 \pm 5.68$ & $22.30 \pm 0.73$ & $<0.01$ \\
Height $(\mathrm{cm})$ & $161.69 \pm 6.58$ & $163.90 \pm 6.18$ & 0.33 \\
Weight $(\mathrm{kg})$ & $60.29 \pm 8.76$ & $56.40 \pm 7.61$ & 0.13 \\
\hline Body mass index $\left(\mathrm{kg} / \mathrm{m}^{2}\right)$ & $22.97 \pm 2.09$ & $20.94 \pm 2.16$ & $<0.01$ \\
\hline
\end{tabular}

Data are expressed as mean \pm standard deviation. 
rotation, i.e., rotation toward (-) and away from $(+)$ the longitudinal axis of the foot during gait; step length; step width; gait velocity; cadence; gait length, i.e., the length of COP movement during the entire stance phase; single support line, i.e., the length of COP movement during single-leg support; anterior-posterior sway, i.e., the longitudinal distance between the COP intersection point and the midpoint connecting the heels of both feet; and lateral symmetry, i.e., the horizontal distance from the COP intersection point to the midpoint of the horizontal line connecting the COP lines of both feet. Negative and positive values for lateral symmetry indicate shifts to the left and right, respectively. ${ }^{27}$

\section{Procedure}

All participants in both groups were asked to walk barefoot, at a comfortable walking pace, over the 3-meter-long pressure plate 10 times to familiarize themselves with the equipment. ${ }^{28}$ Participants then completed 10 trials at a selfselected pace, with the gait parameters recorded in the middle of the eighth trial. ${ }^{29}$ Anterior-posterior sway and lateral symmetry were calculated automatically by WinPDM software (ver. 1.2.2; Mitel, Ottawa, Canada). To calculate the symmetry of the gait parameters (step length, foot rotation, length of gait, and single support line), the symmetry index, which is the most sensitive measurement of gait symmetry based on spatiotemporal parameters, was used. ${ }^{29}$ The formula for calculating the symmetry index is $\left(\mathrm{X}_{\text {side1- }}\right.$ $\left.\mathrm{X}_{\text {side2 }}\right) /\left[0.5 \times\left(\mathrm{X}_{\text {side1 }}+\mathrm{X}_{\text {side2 }}\right)\right] \times 100 .{ }^{30}$ The symmetry index is expressed as a percentage, with a value of zero indicating perfect symmetry. ${ }^{30}$

\section{Statistical analysis}

The weight, velocity, cadence, and lateral symmetry variables were parametric; all other variables were nonparametric. To compare anthropometric data, step width, stride length, velocity, cadence, and gait symmetry between the elderly and young groups, independent $t$-tests and the Mann-Whitney $U$ test were used for normally and nonnormally distributed variables, respectively. The level of statistical significance was set at $p<0.05$. SPSS software (version 21.0; SPSS Inc., Chicago, IL, USA) was used for the statistical analysis.

\section{RESULTS}

The normality of the dependent variables was assessed using the Kolmogorov-Smirnov test. If the data represents a normal distribution, perform an independent $t$-tests; otherwise, perform a Mann-Whitney U test to compare anthro- pometric data, step width, stride length, velocity, cadence, and gait symmetry between the elderly and young groups.

The body mass index (BMI) was higher in the elderly group compared to the young group (mean difference $=2.02$ $\mathrm{kg} / \mathrm{m}^{2}, 95 \%$ confidence interval $[\mathrm{CI}]=0.72-3.32 \mathrm{~kg} / \mathrm{m}^{2}, p<$ 0.01) (Table 1).

There was no significant group difference in velocity, step width, or stride length $(p>0.05)$. Furthermore, there was no significant group difference in the symmetry of step length, foot rotation, length of gait, single support line, or lateral symmetry $(p>0.05)$. The elderly group showed higher cadence than the young group (mean difference $=8.98$ steps/ min, 95\% CI=3.56-14.4 steps/min, $p<0.01$ ), and also had a higher anterior-posterior sway value (mean difference $=5.88$ $\mathrm{mm}, 95 \% \mathrm{CI}=0.40-11.36 \mathrm{~mm}, p=0.04)($ Table 2$)$.

\section{DISCUSSION}

The purpose of this study was to compare gait parameters and symmetry between healthy elderly and healthy young individuals. Significant group differences were seen in cadence and anterior-posterior sway. However, interestingly, the healthy elderly people, who were screened for pathologies, demonstrated similar walking velocity, step width, stride length, and gait symmetry to those of young people. These findings suggest that elderly people who are healthy and fit may be able to avoid deterioration of gait parameters and symmetry with age.

There was no significant group difference in walking velocity, step width, or stride length between the healthy elderly and healthy young groups in this study. In accordance with this result, a previous study demonstrated similar preferred walking speed, stride length, and stride velocity during the straight walking and turning phases of the 6minute walk test between fit older and younger participants. ${ }^{31,32}$ However, in contrast to the current results, another study reported shorter stride length and slower gait speed in healthy older people than younger people during walking at a self-selected speed. ${ }^{18}$ A systematic review indicated that match-speed analysis would be required to analyze stride length and gait speed independently. The review also indicated that elderly people can walk at the same speed as young people, albeit with an increased contribution of the hip and decreased contribution of the ankle. ${ }^{33}$ The current study found no significant group difference in stride length in statistically similar speed between groups. Although we did not analyze the kinematics and kinetics of the hip, knee, or ankle, altered hip kinetics might remain similar in velocity, step width, and stride length due to compensation for the decreased contribution of the ankle 
Table 2. Comparison of gait parameters and symmetry value between young and elderly group during gait

\begin{tabular}{|c|c|c|c|}
\hline Parameters & Elderly & Young & $p$ \\
\hline Step width $(\mathrm{cm})$ & $9.79 \pm 2.57$ & $10.70 \pm 3.21$ & 0.37 \\
\hline Stride length $(\mathrm{cm})$ & $110.67 \pm 11.41$ & $110.0 \pm 10.24$ & 0.91 \\
\hline Velocity $(\mathrm{km} / \mathrm{h})$ & $3.75 \pm 0.42$ & $3.60 \pm 0.39$ & 0.23 \\
\hline Cadence (steps/min) & $112.58 \pm 7.29$ & $103.60 \pm 10.46$ & $<0.01 *$ \\
\hline Symmetry of step length (\%) & $3.99 \pm 3.83$ & $3.26 \pm 2.69$ & 0.65 \\
\hline Symmetry of foot rotation (\%) & $88.83 \pm 97.01$ & $71.09 \pm 72.71$ & 0.54 \\
\hline Symmetry of length of gait line of COP (\%) & $2.68 \pm 2.75$ & $2.61 \pm 2.55$ & 0.97 \\
\hline Symmetry of single support line of COP (\%) & $5.29 \pm 3.84$ & $5.03 \pm 3.12$ & 0.89 \\
\hline Anterior/posterior position of $\mathrm{COP}(\mathrm{mm})$ & $141.74 \pm 9.01$ & $135.86 \pm 8.85$ & $0.04 *$ \\
\hline Lateral symmetry of COP (mm) & $0.65 \pm 3.95$ & $0.33 \pm 3.02$ & 0.77 \\
\hline
\end{tabular}

Data are expressed as mean \pm standard deviation.

Abbreviations: COP, center of pressure.

as similar with a previous study. ${ }^{33}$

There was no significant group difference in symmetry of step length, foot rotation, or COP parameters in this study. Similarly, a previous study demonstrated no significant difference in symmetry of step length, foot rotation, or gait length between older patients with unilateral knee osteoarthritis and healthy older controls, although there was a difference in lateral symmetry. ${ }^{34}$ Healthy elderly participants showed reduced lateral symmetry $(0.65 \mathrm{~mm})$ relative to healthy older participants $(-2.6 \mathrm{~mm})$ in a previous study. ${ }^{34}$ These differences in results might reflect differences in the study inclusion criteria; the healthy elderly participants in the current study had no musculoskeletal pain in the lower back or lower limbs, and no medical history of depression, hypertension, dementia, diabetes, or osteoarthritis, whereas in the previous study the elderly participants only had to be free of knee pain during gait to be classified as healthy. Regarding symmetry of step length, in a small previous study (10 subjects per group), healthy older people who were able to walk for at least 20 minutes with no orthopedic abnormalities, and who had a history of at least one fall within the previous 2 years, showed greater asymmetry in step length during preferred-speed walking than did young people. ${ }^{7}$ The current study recruited elderly participants with no lifetime fall history who were able to walk for at least 1 hour, and go up and down stairs, without pain. The participants in the current study were thus more fit than those in the previous study, which may explain the similar symmetry index values between the elderly and young groups. However, it is difficult to conclude based on these data alone that healthy elderly people walk more symmetri- cally than those who are less healthy; future research should compare gait symmetry according to fitness levels in healthy older populations.

High-functioning healthy older adults were able to walk with relatively high cadence ( $>100$ steps $/ \mathrm{min})$ in a freeliving setting, ${ }^{35}$ and a narrative review suggested that a cadence of $>100 \mathrm{steps} / \mathrm{min}$ is a reasonable threshold for defining moderate-intensity walking in healthy adults. ${ }^{36}$ Another previous study reported higher cadence (111.63 step/min) in a healthy older group with no pathologies compared to a young group (106.84 step/min). ${ }^{11}$ In the current study, the healthy elderly group (112.58 step/min) showed significantly higher cadence than the young group (103.60 step/min). Although the healthy elderly participants showed high cadence ( $>100$ steps/min) both in previous studies and the current one, this was an unexpected result. Elderly people may increase both cadence and stride width to compensate for insufficient mediolateral stability when walking along a narrow pathway. Higher cadence in elderly participants during gait might reflect a compensatory strategy. ${ }^{11}$ However, the ability of higher cadence to improve mediolateral stability during walking requires further investigation.

The variation in anterior-posterior sway among our elderly group $(141.74 \mathrm{~mm})$ was higher than that among the younger group (135.86 mm). This result is consistent with previous findings, which demonstrated greater variability in anterior-posterior sway during the pre-swing phase of gait in older compared to younger participants. ${ }^{37}$ According to a previous study, anterior-posterior sway may increase under unstable conditions, for example when the eyes are closed. ${ }^{38}$ 
Our elderly group showed more anterior-posterior instability during gait compared to the young group. This increase in anterior-posterior sway could be associated with fall risk, as suggested by a previous study. ${ }^{37}$ In addition, higher BMI could have a greater influence on anteriorposterior COP sway in elderly than young people, because increased body weight and waist circumference increased anterior sway in a previous study. ${ }^{39}$

The first limitation of this study was that the gait measurement duration was short. When measured over more than 30 minutes, gait asymmetry may be greater, and walking stability lower, in older adults..$^{23}$ The second limitation was that the gait parameters, COP variables, and gait symmetry findings may not generalize to individuals not aged 50-70 years or in their 20 s (i.e., the ages of our older and younger groups, respectively). Thirdly, we did not consider the effects of strength, flexibility and alignment such as genu valgus or varus on gait symmetry. Previous studies have demonstrated that asymmetry of the knee extensors and iliotibial band flexibility can induce asymmetry of gait and foot alignment, ${ }^{19,40}$ further research is needed on this. Lastly, current study did not measure the joint kinematics with spatio-temporal parameter, so future study would be needed for young and older adults.

\section{CONCLUSIONS}

In this study, healthy elderly people with no medical history of depression, hypertension, dementia, diabetes, osteoarthritis, or musculoskeletal pain during walking, or when going up or down stairs, had similar walking velocity, step width, stride length, and gait symmetry to healthy young people; differences were only seen in higher cadence and anterior-posterior sway in elderly than young group. Based on these findings, clinicians should inform elderly people about the importance of maintaining fitness and managing the musculoskeletal system to prevent deterioration of gait.

\section{Key Points}

Question Do healthy elderly individuals without depression, hypertension, dementia, diabetes as well as musculoskeletal pain have similar gait spatiotemporal parameters and symmetry to healthy young people?

Findings There was no significant difference in walking velocity, step width, stride length, or gait symmetry between healthy elderly and healthy young people. Elderly people showed higher cadence and greater anterior-posterior sway compared to young people.
Meaning These findings suggest that if elderly people are healthy and fit, deterioration of gait parameters and symmetry can be avoided.

\section{Article information}

Conflict of Interest Disclosures: None.

Funding/Support: This work was supported by the 2020

Research-Year Grant of Jeonju University.

Acknowledgment: None.

Ethic Approval: This study was approved by the Jeonju University Campus Human Studies Committee.

\section{REFERENCES}

1. Laufer Y. Effect of age on characteristics of forward and backward gait at preferred and accelerated walking speed. J Gerontol A Biol Sci Med Sci. 2005;60(5):627-632.

2. Aboutorabi A, Arazpour M, Bahramizadeh M, Hutchins SW, Fadayevatan R. The effect of aging on gait parameters in able-bodied older subjects: a literature review. Aging Clin Exp Res. 2016;28(3):393-405.

3. Cromwell RL, Newton RA. Relationship between balance and gait stability in healthy older adults. J Aging Phys Act. 2004;12(1):90-100.

4. Chamberlin ME, Fulwider BD, Sanders SL, Medeiros JM. Does fear of falling influence spatial and temporal gait parameters in elderly persons beyond changes associated with normal aging? J Gerontol A Biol Sci Med Sci. 2005;60(9):1163-1167.

5. Kerrigan DC, Todd MK, Della Croce U, Lipsitz LA, Collins JJ. Biomechanical gait alterations independent of speed in the healthy elderly: evidence for specific limiting impairments. Arch Phys Med Rehabil. 1998;79(3):317322.

6. Ko S, Ling SM, Winters J, Ferrucci L. Age-related mechanical work expenditure during normal walking: The baltimore longitudinal study of aging. J Biomech. 2009; 42(12):1834-1839.

7. Grecco LA, Zanon N, Sampaio LM, Oliveira CS. A comparison of treadmill training and overground walking in ambulant children with cerebral palsy: Randomized controlled clinical trial. Clin Rehabil. 2013;27(8):686696.

8. Hollman JH, McDade EM, Petersen RC. Normative spatiotemporal gait parameters in older adults. Gait Posture. 2011;34(1):111-118.

9. Silder A, Heiderscheit B, Thelen DG. Active and passive contributions to joint kinetics during walking in older adults. $J$ Biomech. 2008;41(7):1520-1527. 
10. Winter DA, Patla AE, Frank JS, Walt SE. Biomechanical walking pattern changes in the fit and healthy elderly. Phys Ther. 1990;70(6):340-347.

11. Shkuratova N, Morris ME, Huxham F. Effects of age on balance control during walking. Arch Phys Med Rehabil. 2004;85(4):582-588.

12. Bautmans I, Jansen B, Van Keymolen B, Mets T. Reliability and clinical correlates of $3 \mathrm{~d}$-accelerometry based gait analysis outcomes according to age and fall-risk. Gait Posture. 2011;33(3):366-372.

13. Yogev G, Plotnik M, Peretz C, Giladi N, Hausdorff JM. Gait asymmetry in patients with parkinson's disease and elderly fallers: when does the bilateral coordination of gait require attention? Exp Brain Res. 2007;177(3):336346.

14. Moe-Nilssen R, Helbostad JL. Estimation of gait cycle characteristics by trunk accelerometry. J Biomech. 2004; 37(1):121-126.

15. Kobayashi H, Kakihana W, Kimura T. Combined effects of age and gender on gait symmetry and regularity assessed by autocorrelation of trunk acceleration. $J$ Neuroeng Rehabil. 2014;11:109.

16. Peterson DS, Martin PE. Effects of age and walking speed on coactivation and cost of walking in healthy adults. Gait Posture. 2010;31(3):355-359.

17. Hahn ME, Chou LS. Age-related reduction in sagittal plane center of mass motion during obstacle crossing. $J$ Biomech. 2004;37(6):837-844.

18. Ostrosky KM, VanSwearingen JM, Burdett RG, Gee Z. A comparison of gait characteristics in young and old subjects. Phys Ther. 1994;74(7):637-644.

19. Laroche DP, Cook SB, Mackala K. Strength asymmetry increases gait asymmetry and variability in older women. Med Sci Sports Exerc. 2012;44(11):2172-2181.

20. Allet L, Armand S, de Bie RA, et al. Gait alterations of diabetic patients while walking on different surfaces. Gait Posture. 2009;29(3):488-493.

21. Niedenthal PM, Barsalou LW, Winkielman P, KrauthGruber S, Ric F. Embodiment in attitudes, social perception, and emotion. Pers Soc Psychol Rev. 2005; 9(3): 184-211.

22. Boa Sorte Silva NC, Gill DP, Nagamatsu LS, Owen AM, Petrella RJ. Systolic blood pressure dipping may be associated with mobility impairment and brain volume in community-dwelling older adults: an exploratory study. Exp Gerontol. 2020;141:111100.

23. Wong DW, Lam WK, Lee WC. Gait asymmetry and variability in older adults during long-distance walking: implications for gait instability. Clin Biomech (Bristol, Avon). 2020;72:37-43.
24. Virmani T, Gupta H, Shah J, Larson-Prior L. Objective measures of gait and balance in healthy non-falling adults as a function of age. Gait Posture. 2018;65:100105.

25. Sadeghi H, Allard P, Prince F, Labelle H. Symmetry and limb dominance in able-bodied gait: a review. Gait Posture. 2000;12(1):34-45.

26. Faude O, Donath L, Roth R, Fricker L, Zahner L. Reliability of gait parameters during treadmill walking in community-dwelling healthy seniors. Gait Posture. 2012;36(3):444-448.

27. Yoo SD, Kim HS, Lee JH, et al. Biomechanical parameters in plantar fasciitis measured by gait analysis system with pressure sensor. Ann Rehabil Med. 2017;41(6): 979-989.

28. Byrne JE, Stergiou N, Blanke D, Houser JJ, Kurz MJ, Hageman PA. Comparison of gait patterns between young and elderly women: an examination of coordination. Percept Mot Skills. 2002;94(1):265-280.

29. Wearing SC, Reed LF, Urry SR. Agreement between temporal and spatial gait parameters from an instrumented walkway and treadmill system at matched walking speed. Gait Posture. 2013;38(3):380-384.

30. Patterson KK, Gage WH, Brooks D, Black SE, McIlroy WE. Evaluation of gait symmetry after stroke: a comparison of current methods and recommendations for standardization. Gait Posture. 2010;31(2):241-246.

31. Mariani B, Hoskovec C, Rochat S, Büla C, Penders J, Aminian K. 3d gait assessment in young and elderly subjects using foot-worn inertial sensors. $J$ Biomech. 2010;43(15):2999-3006.

32. Shi L, Duan F, Yang Y, Sun Z. The effect of treadmill walking on gait and upper trunk through linear and nonlinear analysis methods. Sensors (Basel). 2019;19(9).

33. Boyer KA, Johnson RT, Banks JJ, Jewell C, Hafer JF. Systematic review and meta-analysis of gait mechanics in young and older adults. Exp Gerontol. 2017;95:63-70.

34. Kim SH, Park KN. Lateral symmetry of center of pressure during walking in patients with unilateral knee osteoarthritis. Physical Therapy Korea. 2021;28(1):7783.

35. Tudor-Locke C, Barreira TV, Brouillette RM, Foil HC, Keller JN. Preliminary comparison of clinical and freeliving measures of stepping cadence in older adults. $J$ Phys Act Health. 2013;10(8):1175-1180.

36. Tudor-Locke C, Han H, Aguiar EJ, et al. How fast is fast enough? Walking cadence (steps $/ \mathrm{min}$ ) as a practical estimate of intensity in adults: a narrative review. $\mathrm{Br} J$ Sports Med. 2018;52(12):776-788.

37. Bizovska L, Svoboda Z, Kutilek P, Janura M, Gaba A, 
Kovacikova Z. Variability of centre of pressure movement during gait in young and middle-aged women. Gait Posture. 2014;40(3):399-402.

38. Opala-Berdzik A, Błaszczyk JW, Bacik B, et al. Static postural stability in women during and after pregnancy: a prospective longitudinal study. PLoS One. 2015;10(6): e0124207.

39. Corbeil P, Simoneau M, Rancourt D, Tremblay A,
Teasdale N. Increased risk for falling associated with obesity: mathematical modeling of postural control. IEEE Transactions on Neural Systems and Rehabilitation Engineering. 2001;9(2):126-136.

40. Kim SJ, You KJ, Jung DY. Between-side comparisons of iliotibial band flexibility and the tibial torsion angle in subjects with an asymmetric hallux valgus angle. $J$ Musculoskelet Sci Technol. 2018;2(1):11-15. 